\title{
Rising School Enrollment and Declining HIV and Pregnancy Risk Among Adolescents in Rakai District, Uganda, 1994-2013
}

\author{
John S. Santelli ${ }^{1}$ - Sanyukta Mathur ${ }^{1} \cdot$ Xiaoyu Song ${ }^{2}$ - Tzu Jung Huang ${ }^{2} \cdot$ Ying Wei $^{2}$. \\ Tom Lutalo $^{3} \cdot$ Fred Nalugoda $^{3} \cdot$ Ron Gray $^{4} \cdot$ David Serwadda $^{3}$
}

Published online: 8 May 2015

(C) Springer International Publishing 2015

\begin{abstract}
Background Poverty, family stability, and social policies influence the ability of adolescents to attend school. Likewise, being enrolled in school may shape an adolescent's risk for HIV and pregnancy. We identified trends in school enrollment, factors predicting school enrollment (antecedents), and health risks associated with staying in or leaving school (consequences).

Methods Data from the Rakai Community Cohort Study (RCCS) were examined for adolescents $15-19$ years $(n=21$, 735 person-rounds) from 1994 to 2013 . Trends, antecedents, and consequences were assessed using logistic and linear regression with robust variance estimation. Qualitative data were used to explore school leaving among HIV+ and HIVyouths (15-24 years).

Results School enrollment and socioeconomic status (SES) rose steadily from 1994 to 2013 among adolescents; orphan-
\end{abstract}

John S. Santelli

js2637@columbia.edu

Sanyukta Mathur

sm2892@columbia.edu

Xiaoyu Song

xs2148@columbia.edu

Tzu Jung Huang

th2455@columbia.edu

Ying Wei

yw2148@columbia.edu

Tom Lutalo

tlutalo@rhsp.org

Fred Nalugoda

fnalugoda@rhsp.org hood declined after availability of antiretroviral therapy. Antecedent factors associated with school enrollment included age, SES, orphanhood, marriage, family size, and the percent of family members $<20$ years. In qualitative interviews, youths reported lack of money, death of parents, and pregnancy as primary reasons for school dropout. Among adolescents, consequences associated with school enrollment included lower HIV prevalence, prevalence of sexual experience, and rates of alcohol use and increases in consistent condom use. Young women in school were more likely to report use of modern contraception and never being pregnant. Young men in school reported fewer recent sexual partners and lower rates of sexual concurrency.

Conclusions Rising SES and declining orphanhood were associated with rising school enrollment in Rakai. Increasing school enrollment was associated with declining risk for HIV and pregnancy.
Ron Gray

rgray@jhsph.edu

David Serwadda

dserwada@infocom.co.ug

Heilbrunn Department of Population \& Family Health, Mailman School of Public Health, Columbia University, 60 Haven Avenue B-2, New York, NY 10032, USA

2 Department of Biostatistics, Mailman School of Public Health, Columbia University, 722 West 168th Street, 6th Floor, New York, NY 10032, USA

3 Rakai Health Sciences Program, Uganda Virus Research Institute, Nakiwogo Road, P.O. Box 49, Entebbe, Uganda

4 Department of Epidemiology, Bloomberg School of Public Health, Johns Hopkins University, 615 N Wolfe St \#5041, Baltimore, MD 21205, USA 
Keywords Education - Orphanhood - Socioeconomic status · HIV risk · Sibship · Gender

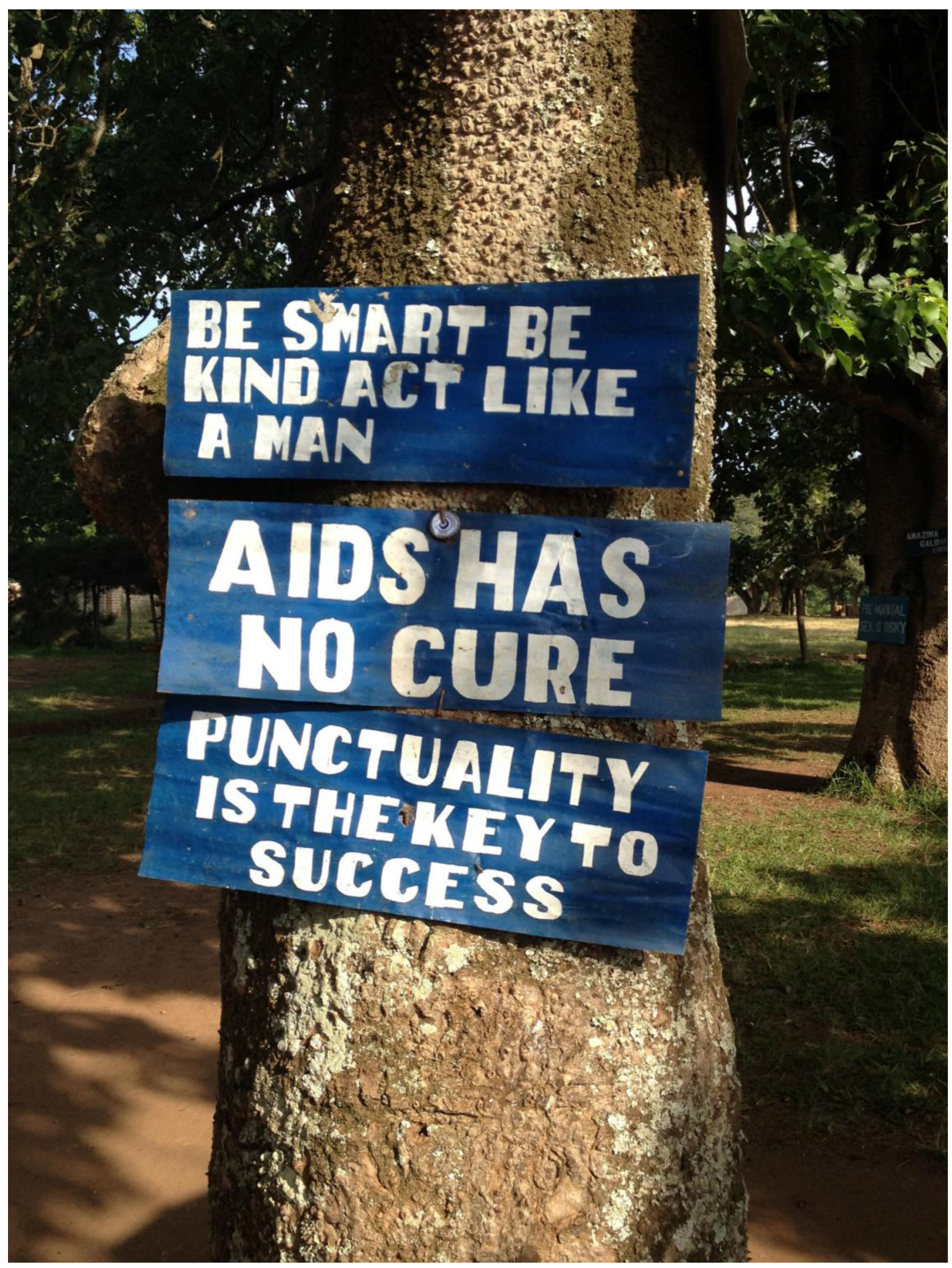




\section{Introduction}

Education has a powerful influence on health across the lifespan (Caldwell and Caldwell 1986; Mavedzenge et al. 2013). Education is associated with improved health practices, social and economic empowerment, and health literacy. Mothers' educational attainment is associated with improved health for children, adolescents, and women (Lloyd 2005; Cleland 2010). Among young people, education is a protective factor, with greater educational achievement, connectedness to school, and continuing school attendance associated with lower rates of health risk behaviors and lower rates of adolescent and unintended pregnancy (Bernat and Resnick 2006; Kirby et al. 2007; Mmari and Blum 2009). In developed countries, children and adolescents who are doing well in school academically or socially, who feel connected to the school, and who have aspirations for the future are more likely to have greater educational attainment and fewer health risk behaviors (Bernat and Resnick 2006; Kirby et al. 2007). In developing nations, young people who have access to primary and secondary schools demonstrate higher educational and occupational attainment (Lloyd 2005; Mmari and Blum 2009). In both settings, youths, who have parents and families who are supportive of education, are likely to do better in terms of health, education, and work. Rising educational attainment across developing and developed nations is associated with improving child health status (Gakidou et al. 2010); education may also improve health equity (Mavedzenge et al. 2013).

Social and structural factors influence access to education at multiple levels. The wealth of nations and individual families influences access to education; education is commonly perceived as a gateway to social advancement by families and essential for economic and human development by political leaders. Thus, the distribution of educational resources is influenced by income distribution and the stage of nation's economic development (Deininger 2003). Within the family, financial insecurity and factors such as sibship size (many children competing for limited family resources) may influence the ability of youths to continue education (Cyrus Chu et al. 2007). Orphanhood among youths in Sub-Saharan Africa (SSA) (often because of HIV infection among parents) may be associated with truncation of schooling (Birdthistle et al. 2009). Poor families may be unable to afford school fees and uniforms. Schools may be far away, or there might be a scarcity of qualified teachers. Many direct and indirect costs of schooling can often present barriers to school enrollment, particularly in poor communities. In Uganda, rising school enrollment is linked to the national policy of Universal Primary Education (UPE) inaugurated in 1997 which abolished tuition fees and increased educational access for children and adolescents, particularly young women (Deininger 2003).

Increases in school enrollment profoundly shape social developmental transitions among youths such as the timing of sexual debut, entrance into marriage, and initiation of childbearing (Lloyd 2005). As youths remain in school, they delay workforce entrance and family formation and may delay initiation of sexual intercourse and childbearing (Lloyd 2005). Increased access to education for young women may be a particularly powerful influence on these social transitions, as education creates new and expanding career opportunities which are alternatives to family formation and childbearing during the teen years. Changes in these social transitions may influence risk for HIV and other sexually transmitted infections (STIs). For example, youths may delay entrance into marriage, initiate sexual intercourse premaritally, and experience increases in lifetime sexual partners.

HIV/AIDS has had a profound impact on youths (1524 years) in SSA. Risk factors for HIV infection include multiple sexual partners, sexual concurrency, commercial sex work, failure to use barrier protection, and STI; power dynamics within relationships; and biological factors such as pubertal timing and circumcision (Mmari and Blum 2009). In our prior research with youths in the Rakai District of southwestern Uganda, school enrollment was associated with reduced HIV acquisition among youths (Santelli et al. 2013) and rising school enrollment was associated with declining rates of HIV acquisition among adolescent women (but not for adolescent men or young adults) (Santelli et al. 2015).

Rakai District has seen rising school enrollment and the implementation of multiple HIV treatment and prevention programs over the past 20 years (Gray et al. 2007). The Rakai Youth Project (RYP) has explored pathways for HIV acquisition among youths in the Rakai District of southwestern Uganda. Research has been guided by a proximate determinants theoretical framework which emphasized the critical roles of underlying determinants such as developmental transitions, social context, and prevention policies in influencing proximate determinants such as HIV risk behaviors, biological factors, and HIV infection (Fig. 1, Boerma and Weir 2005; Pettifor et al. 2008). Development transitions and social context include life goals, school enrollment, relationship formation, marriage, and childbearing.

In this study, we first explored possible antecedents (i.e., potential predictors) of rising school enrollment among adolescents in Rakai, including household socioeconomic status (SES) and orphanhood. Our analyses exploring antecedents examined both the cross-sectional association of potential risk factors with school enrollment and the ecological association between trends in antecedents and school enrollment from 


\section{Distal Factors}

Social and Contextual Individual Psychosocial Factors

\begin{tabular}{|l|}
\hline Social Context \\
Family influences \\
Migration \& mobility \\
Peer pressure \\
Partner influences \\
HIV - related stigma \\
Trading villages
\end{tabular}

HIV Policies \& Programs

\begin{tabular}{|l|l|l}
\hline Zero grazing & $\begin{array}{l}\text { Perceived risk of HIV } \\
\text { ARC/abstinence } \\
\text { ARV treatment optimism }\end{array}$ \\
$\begin{array}{l}\text { Access to male } \\
\text { circumcision }\end{array}$ & $\begin{array}{l}\text { Perceived effectiveness of male } \\
\text { circumcision }\end{array}$ \\
\hline
\end{tabular}

Fig. 1 Biopsychosocial framework for Rakai Youth Project

1994 to 2013 . We then used a similar approach to examine possible consequences of rising school enrollment on risk factors for HIV infection and pregnancy and social transitions such as sexual initiation, marriage, and pregnancy. Both sets of analyses were guided by a conceptual model focusing on school enrollment (Fig. 2). We also used qualitative methods to illuminate the relationship between poverty, orphanhood,

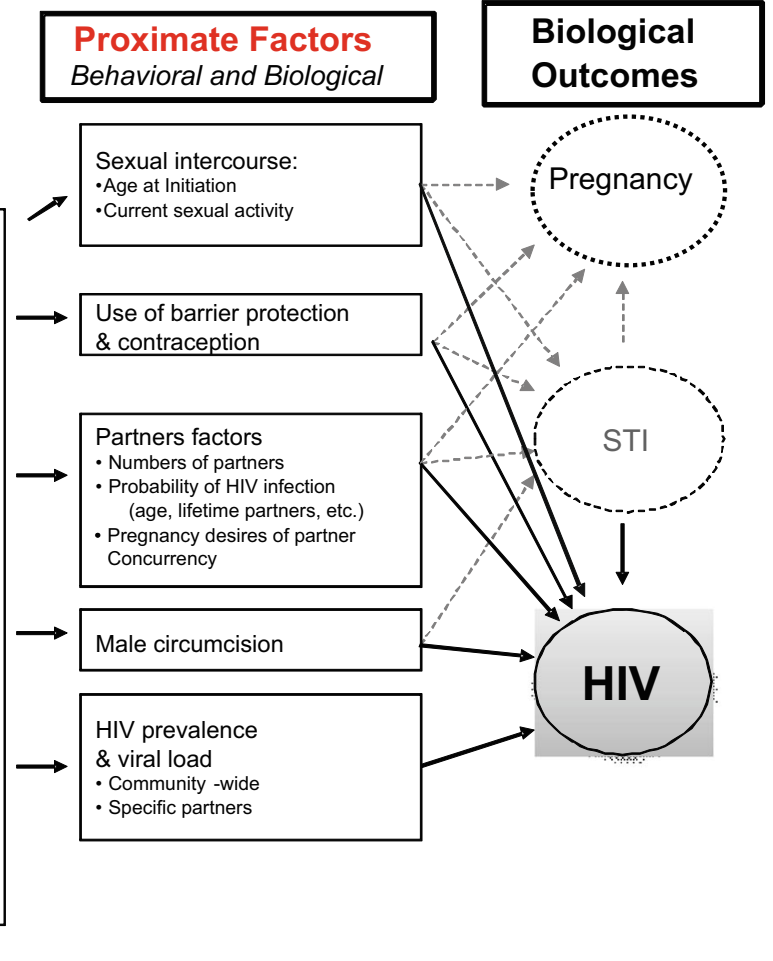

and school enrollment and dropout. We hypothesized that (1) rising levels of household SES and family factors such as orphanhood and sibship size would be associated with rising school enrollment and (2) school enrollment would be associated with delay in social transitions and reductions in risk for HIV and pregnancy. We explored how HIV risk is shaped by education, social transitions, and public policies.
Fig. 2 Antecedents and consequences of school enrollment, Rakai District, Uganda

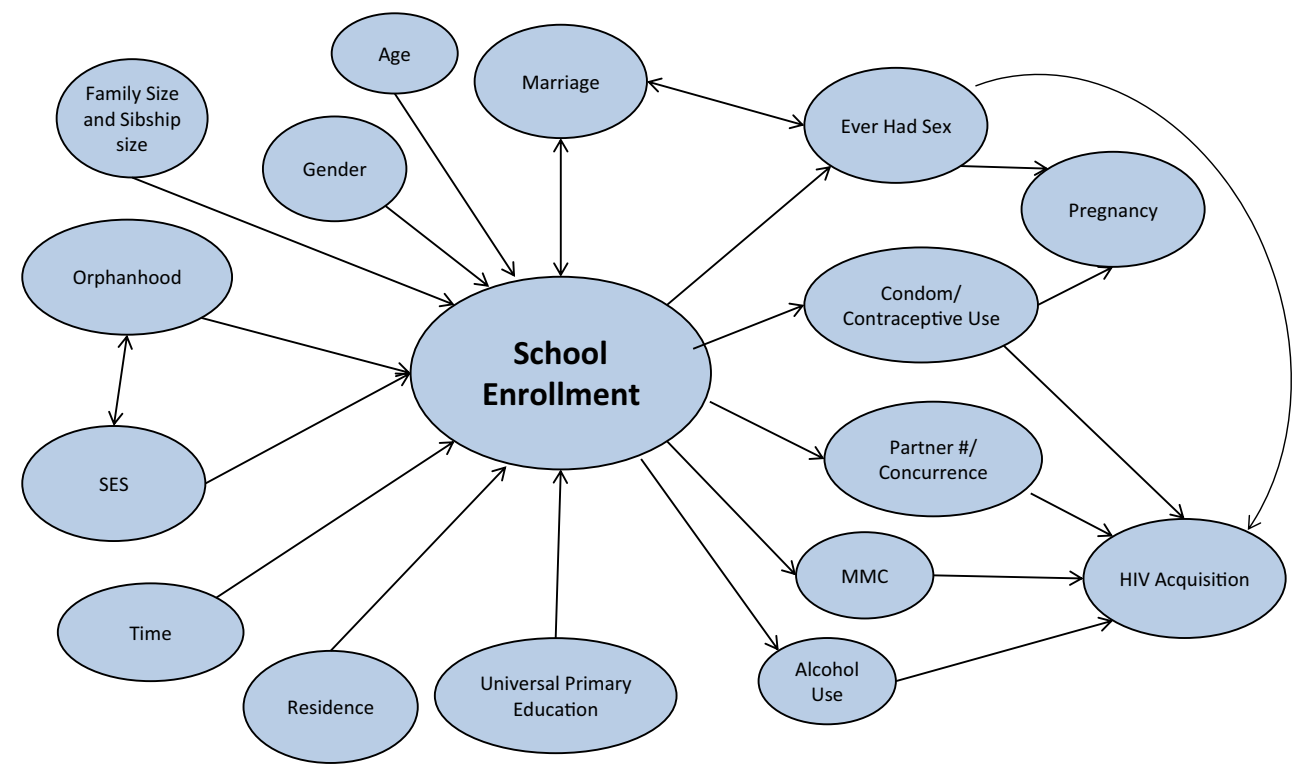




\section{Methods}

\section{Study Design and Setting}

RYP has used a mixed method study design and relied on longitudinal cohort data from the Rakai Community Cohort Study (RCCS) and on in-depth qualitative interviews with youths nested within the RCCS. Ethical approvals were obtained from Uganda National Council for Science and Technology and institutional review boards (IRBs) at the Uganda Virus Research Institute, Columbia University, and Johns Hopkins University and Western IRB. Research was performed in accordance with the ethical standards of the Declaration of Helsinki and the Belmont Report.

Over time, the Rakai Health Sciences Program has progressively implemented HIV prevention and treatment programs including interventions to prevent HIV infection via STI treatment in 1994, prevention of maternal to child transmission in 2000, anti-retroviral treatment (ART) in 2004, and male medical circumcision (MMC) as part of an RCT in 2004 and as a district-wide service in 2007 (Gray et al. 2007).

\section{Qualitative Data Collection and Analysis}

The qualitative data for this study came from an ethnographic case-control study of HIV+ (cases) and HIV- (controls) of young men and women aged 15-24 years (Higgins et al. 2014). In-depth life history interviews were conducted with respondents selected from the RCCS. Interviews occurred between June 2010 and June 2011. Qualitative interviews were structured around four major themes: life goals and transitions, pregnancy and parenthood, HIV risk and avoidance, and sexual relationships and context. We used an iterative process for the qualitative data analysis that involved reading, coding, interpreting, reducing, and displaying the data gathered during the interviews.

\section{Quantitative Data Collection and Study Participants}

We examined quantitative data from the RCCS for adolescents 15-19 years olds living in 28 communities under continuous surveillance between 1994 and 2013 encompassing rounds 1-15; data collection initially was annual, but later rounds took 12-20 months to complete. The RCCS is an open cohort of residents 15-49 years and has been described more extensively elsewhere (Wawer et al. 1998, 1999). Briefly, each round of the RCCS begins with a census of households with data on changes to household composition provided by the head of household. As an open cohort, newly age-eligible 15 years olds are enrolled at each round and older adolescents are recruited to compensate for those lost to follow-up (round 5 was an atypical follow-up round with no new enrollment and was excluded from these analyses.) At each survey round, new and returning cohort participants are consented, interviewed, and asked to provide blood for HIV and STI testing. For minors ( $<18$ years), minor assent and parental/ guardian permission are obtained, and $18+$ years olds provide their own informed consent. At each round, community-wide HIV education, individual and couple HIV counseling and testing, and referral for health care are offered.

A face-to-face interview of each adolescent was conducted in a private location by a same-sex interviewer. Although parents/guardians provided permission for minor adolescent, data from the adolescent were not shared with parents. Questionnaires at each round included questions on demographic, behavioral, reproductive, and health characteristics. The RCCS has achieved over $85 \%$ coverage among all residents. Among consenting participants, $99 \%$ responded to the full questionnaire and over $90 \%$ agreed to specimen collection. Frequent mobility among adolescents contributes to missing data after recruitment into the cohort. Mobility includes travel for school and work and migration outside of the RCCS communities. Over rounds $1-14$ of the survey, $50 \%$ of adolescents surveyed in one round were resurveyed in the next round; an additional $8 \%$ were resurveyed in a later round, and $42 \%$ were lost of follow-up.

\section{Variables}

In this paper, we focus on independent and dependent variables associated with school enrollment which we term antecedents and consequences of school enrollment (Fig. 2). School enrollment was based on a self-reported measure, when a respondent stated that either his or her first or second occupation was "student."

Antecedents included independent factors likely to influence an adolescents' ability to enroll or remain in school such as age of the adolescent, household SES, family size, number of children in the household, and orphanhood. Age was also reported by the adolescent. If data on age were missing, household census data were used to impute age. Data from the household census included residence, orphanhood, socioeconomic status (SES), total household (i.e., family) size, sibship size, and number of children and adults. Area of residence included trading villages (i.e., on a major road) or rural villages. Orphanhood was derived from questions about the current status of each parent (i.e., had mother and/or father died). A SES index was created based on the use of modern building materials to construct the household dwelling; dwellings characterized as high SES had extensive use of modern building materials, and those classified as low SES had little or no use of such materials (Makumbi et al. 2011). We defined sibship size as a categorical variable based on the percentage of household members $<20$ years; four categories were defined based on quartiles of the distribution. Certain social transitions such as ever married can be seen as either an 
antecedent factor (influencing school enrollment) or as a consequence of school enrollment (i.e., staying in school delays entrance into marriage). Thus, delaying marriage may help an adolescent stay in school, or dropping out of school may create social pressure for a young person to marry. For simplicity of presentation, we list ever married as an antecedent factor.

Consequences of school enrollment were dependent factors which could be influenced by school dropout. These included ever had sex, ever being pregnant, current use of a modern family planning method (among women), consistent use of condoms with all sexual partners over the past year, $2+$ sexual partnerships in the past year, sexual concurrency at the time of the interview, alcohol use in the past 30 days, and HIV serostatus. Behaviors were reported by the adolescent during the face-to-face interview. HIV serostatus was based on biological testing. Ever had sex and alcohol use are reported among all adolescents; for all other variables, data are reported for sexually experienced adolescents, those who had ever had sexual intercourse.

\section{Data Analysis}

The quantitative analysis sample consisted of 11,829 personrounds for adolescent women and 9606 person-rounds for men. Person-rounds were total number of observations across all adolescents; most adolescents were included in multiple rounds of the survey and therefore have several observations. Overall, 13,854 person-rounds included adolescent who were sexually experienced; those observations were included in analyses for multiple partners and concurrency. We initially examined characteristics of the sample over time at each round of the survey (see Table 1). We then examined possible antecedent factors as cross tabulations (data found in Table 2). Next, we used multivariate logistic regression controlling for age to identify antecedent factors independently associated with school enrollment (Table 3) (Liang and Zeger 1986). Finally, we used cross tabulations to examine factors which could be consequences of staying in or leaving school (see Table 4).

Regression analyses with robust variance estimation were used to test for changes over time (Freedman 2006). We used, respectively, linear regression, logistic regression, ordinal logistic regression, and multinomial logistic regression with robust variance estimation to test the shifts over time for risk factors that were continuous, binary, ordinal with three categories, and nominal with more than three categories. All analyses were conducted separately for women and men. In the RCCS sample, the mean age of the adolescents changed over time. The mean age rose for women from age 17.1 years in round 1 to 17.5 years in round 10 and then dropped to 17.0 in round 15; similar patterns occurred for men. As such, all regression analyses were adjusted by single year of age or were stratified by age-given shifts over time in the population age structure and because both antecedent and consequent factors were likely to be associated with age.

\section{Results}

Characteristics of the population of adolescents in Rakai changed considerably between 2004 and 2013 (Table 1). The most notable temporal change was a dramatic increase in school enrollment which rose from 25 to $57 \%$ among adolescent women and from 34 to $67 \%$ among adolescent men. School enrollment rose over time for women in at all ages between 15 and 19 years (Fig. 3); similar increases were found for adolescent men (data not shown). The increase in school enrollment for 15 and 16 years olds began as early as 1994 and before UPE was instituted in 1997.

Household SES among adolescent men and women rose steadily over time from 1994 to 2013 (Table 1). In 1994, almost half (49\%) of the households were in the lowest SES category; by 2013, $60 \%$ of households were in the highest SES category. Orphanhood among adolescents was common $(\sim 50 \%)$ in early rounds and declined after $\sim 2004$. This decline coincided with increasing access to ART, declining mortality from AIDS, and declining orphanhood among children in Rakai (Makumbi et al. 2012).

Marriage rates were higher among adolescent women compared to adolescent men and declined substantially among women (47\% in R4 to $25 \%$ in R15) and among men (4.9 to $1.2 \%)$. Reporting ever having had sex increased significantly from rounds 1 to 8 for women and men and then declined significantly from rounds 8 to 15 , with the exception of adolescent women not enrolled in school. The number of family members in households averaged 5.5 over all rounds for women and averaged 6.2 for men; considering single rounds of data collection, household size varied between 5.1 and 6.4 for women and between 5.8 and 6.5 for men. The average number of children and adolescents in families did not change greatly over time.

\section{Quantitative Antecedents of School Enrollment}

We next examined antecedent factors - primarily demographic factors - associated with school enrollment among adolescents (Table 2). School enrollment was sharply different between married and unmarried adolescents; only $0.2 \%$ of married adolescent women were in school, compared to $65 \%$ of unmarried women. Thus, we examined the pattern of associations between antecedent factors and school enrollment among all adolescents and among unmarried adolescents. This pattern of associations was similar across the two groups. As marital status and school enrollment were not available in the early rounds of RCCS data set for rounds $1-4$, we present data for all adolescents in Table 2. 


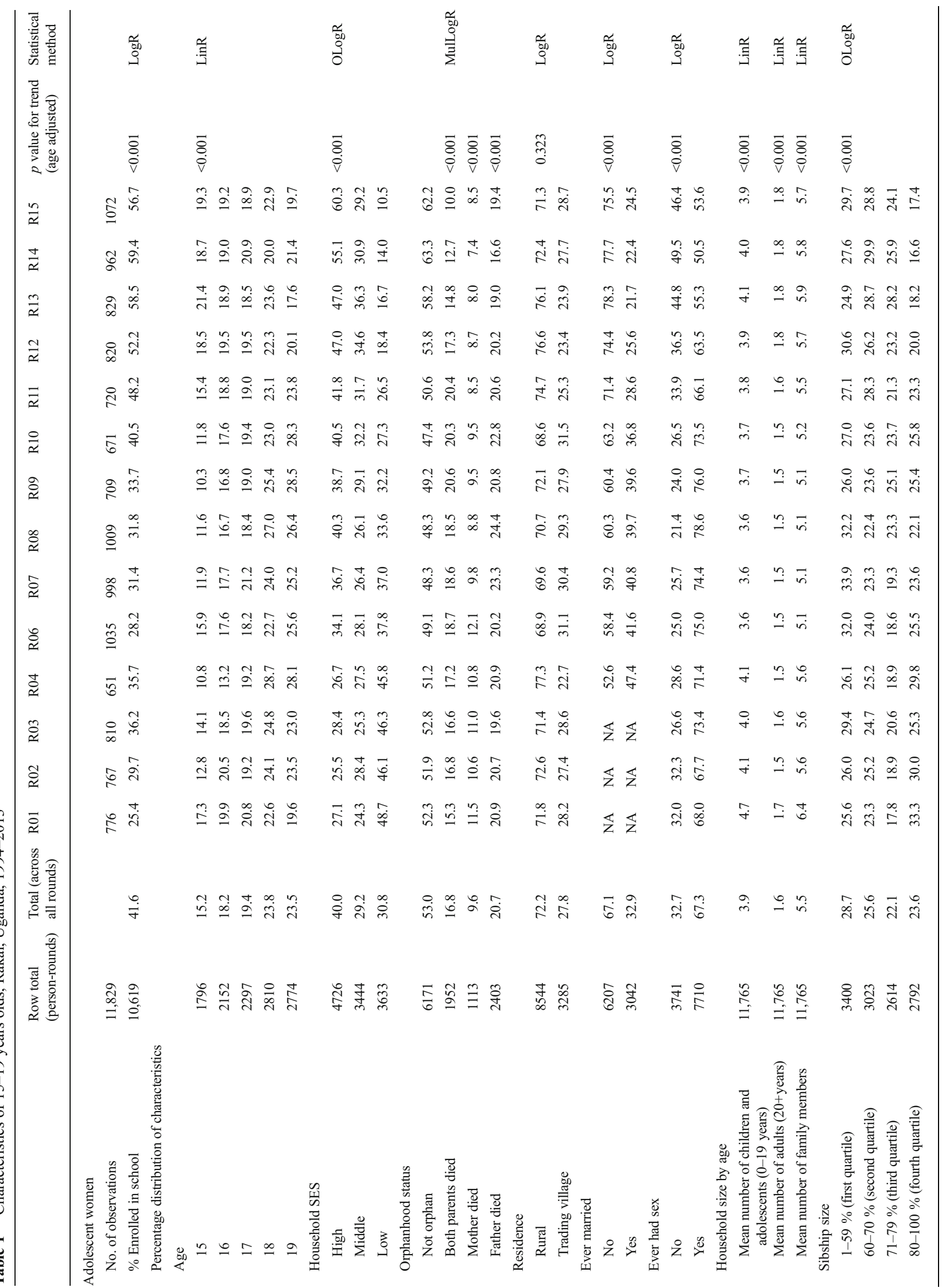




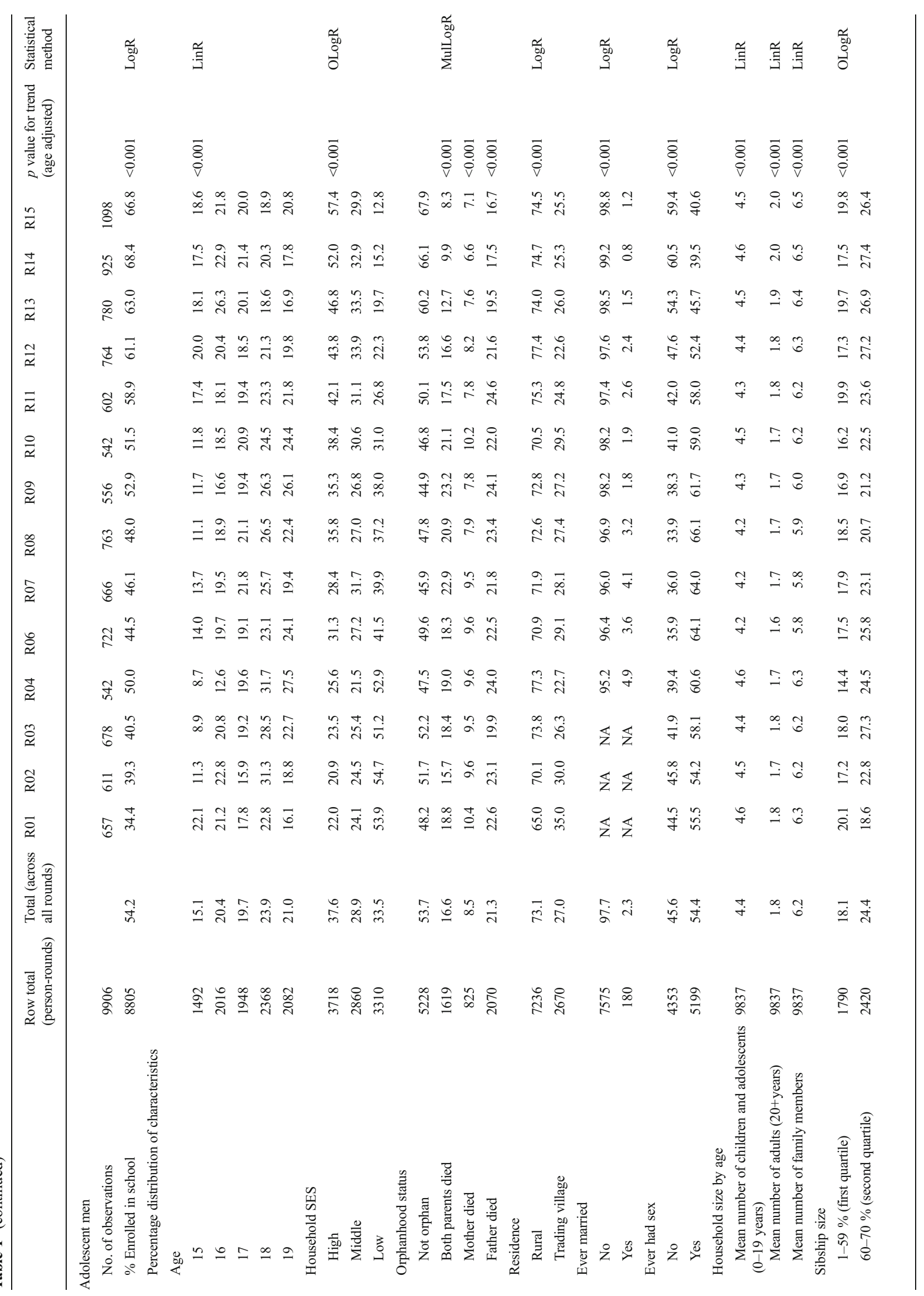




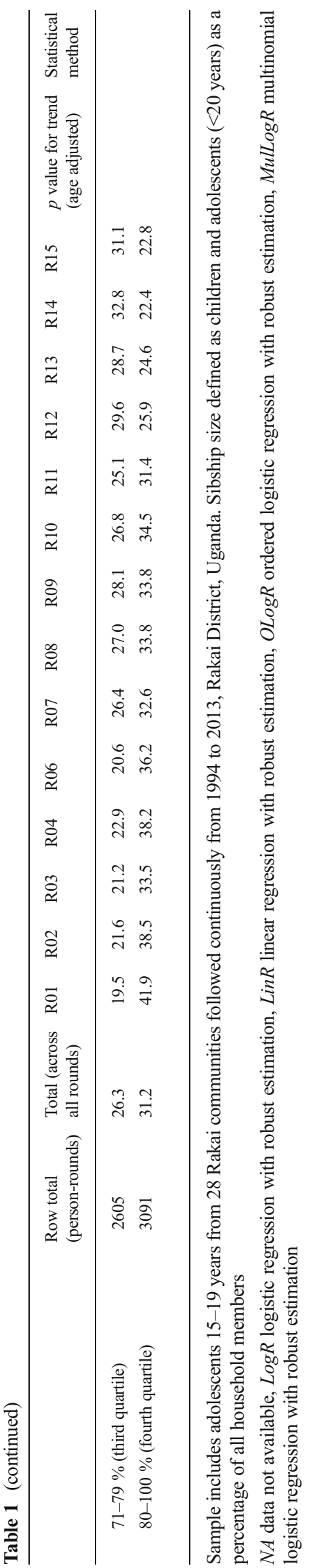

Across all rounds, higher school enrollment for adolescent women was associated with younger age and (after adjusting for age) higher SES, not being an orphan, being never married, and living in a larger family (Table 2). School enrollment was highest among families sibship size in the third quartile (71-79\% of household members under age 20 ) and lowest in the first quartile for adolescent women and the fourth quartile for adolescent men. ${ }^{1}$ School enrollment rose over time for every demographic category in Table 2 , with the exception of ever-married adolescent women.

For adolescent men, we found a similar pattern of school enrollment to that of adolescent women - by age, SES, orphanhood, residence, marital status, family size, and percentage of household members under age 20. Among men, school enrollment rose for every demographic category - with the exception of ever-married men.

The differentials in school enrollment decreased over time between high and low SES and between younger adolescent women and men but increased among older adolescent women and men. In early rounds of RCCS, school enrollment was higher among adolescent men and among high SES adolescents. By round 15, the gender gap for 15-17 years olds had closed; however, the gender gap increased for 18 and 19 years olds. At round 15, school enrollment among women was similar by SES, but among men, higher SES was still associated with higher school enrollment.

Multivariate logistic regression with robust estimation was used to identify independent influences on school enrollment (Table 3). For adolescent women, school enrollment was positively associated with younger age (odds ratio $(\mathrm{OR})=0.6 \mathrm{per}$ year), larger family size ( $\mathrm{OR}=1.13$ per household member), higher SES, and residency in a rural village. Having ever been married $(\mathrm{OR}=0.004)$, having lost both parents $(\mathrm{OR}=0.52)$, or having lost either mother or father $(\mathrm{OR}=0.50$ and 0.66 , respectively) decreased the likelihood of being enrolled in school. A high percentage $\left(80 \%^{+}\right)$of children and adolescents in the household decreased the likelihood that an adolescent woman would be enrolled in school $(\mathrm{OR}=0.77)$.

A similar set of independent predictors was found for school enrollment of adolescent men. However, for adolescent men, residence was not significant, nor was death of a father $(\mathrm{OR}=1.02)$.

\section{Qualitative Antecedents}

Qualitative interviews reinforced findings from the quantitative data. Respondents unanimously reported a desire for education; many aspired to attend secondary school and university. Despite the strong desire for schooling, many respondents in the qualitative sample reported that they were unable to reach their aspirations. Primary challenges to schooling enrollment included financial constraints of the household. The lack of funds for schooling was often the direct result of 
the death of one or more of their parents or, among women, an early pregnancy. One young man noted financial difficulties that hindered his academic achievement, leading to his dropping out of school.

Our father did not have enough money to pay for our education and the school administrator was always sending us home to go and look for school fees. Because of what I was going through I realized that I could no longer compete academically with other students.

Another young man noted, "I dropped out of school after the death of my father and I could not get any more support in terms of school fees." Others reported that a lack of money for school fees resulted from having too many siblings. One young woman noted, "My daddy was educating many children. He told me that since I am a girl, I can forego school so that he is able to pay school fees for my siblings." In such instances, young people expressed a desire not to burden their family or prevent younger siblings from receiving any education, choosing to drop out instead.

\section{Quantitative Consequences of School Enrollment}

School enrollment had a protective effect against risk for HIV and pregnancy among adolescent women and men (Table 4). School enrollment was associated with lower rates of sexual experience and other behaviors associated with HIV infection and pregnancy. Among adolescent women after adjusting for age, school enrollment was associated with lower rates of sexual experience (37 vs. $88 \%$ ), alcohol use in the past 30 days (14 vs. $25 \%$ ), HIV prevalence (1.8 vs. $6.5 \%$ ), and a lower probability of ever having been pregnant (6 vs. $78 \%$ ). School enrollment was related to higher current use of modern contraception ( 45 vs. $22 \%$ ) and a higher rate of consistent condom use with all partners ( 70 vs. $17 \%$ ) School enrollment was not associated with having $2+$ sexual partners in the past year or sexual concurrency.

Among school enrollees, improvements over time were found for seven of eight risk factors for HIV infection and pregnancy; the exception was contraceptive use which rose and then fell. Among non-enrollees, improvements over time occurred in five factors; a more marked change ( $>2$-fold) was found for alcohol use and use of modern contraception, and significant but smaller changes were found for consistent condom use and HIV infection. Ever pregnant among nonenrollees showed a small decline (77.9 to $77.6 \%$ ), which was statistically significant with age adjustment $(p=0.011)$.

School enrollment had a similar set of associations for adolescent men. Enrollment was associated with lower probability of sexual experience (39 vs. $71 \%$ ), alcohol use (34 vs. $16 \%$ ), $2+$ sexual partners in the past year ( 28 vs. $42 \%$ ), and sexual concurrency (6 vs. $11 \%$ ). School enrollees were also more likely to be consistent condom users (70 vs. $50 \%$ ).

\section{Qualitative Consequences}

In the qualitative interviews, we also found a protective effect of school enrollment. Since the ability to attend school was precarious, adolescents who were in currently in school reported postponing social transitions such as marriage and sexual involvement. A young man in school who hoped to study science at the university reported that he was not interested in marrying soon because "my major focus currently is schooling." Another young man reported using "condoms all the time" during sexual encounters to avoid HIV infection and early pregnancy while he was still in school. Young women often noted turning to marriage as a result of dropping out of school, either with the support of their family or by their own choice. One young woman said, "There was no more money for my education so my relatives got me a man to marry."

\section{Discussion}

School enrollment rose considerably from 1994 to 2013 among adolescents in Rakai. Rising SES and declining orphanhood contributed to rising school enrollment. Factors associated with school enrollment included younger age and higher SES, not being an orphan, larger family size, and the proportion of family members under 20 years. In qualitative interviews, youths reported a lack of money, the death of parents, and becoming pregnant as primary reasons for school dropout. Increases in school enrollment were associated with delays in social transitions such as marriage and initiation of sexual intercourse and with declines in risk for HIV and pregnancy among adolescent women. These data suggest that increasing access to school and expanding efforts to retain adolescents in school could provide important improvements in adolescent reproductive health and HIV prevention.

Rising school enrollment appears to be driven by both supply and demand for education. On the supply side, the Ugandan federal mandate for UPE has improved educational access and reduced the wealth bias in educational access (Deininger 2003). Likewise, religious groups such as the Catholic Church and other civil society groups have also expanded the network of private schools in Uganda.

On the demand side, rising SES in Rakai over the past 20 years is presumably tied to rising aspirations for education among parents and young people. With rising SES, education enrollment increased among high and low SES households; differences in school enrollment between poor and non-poor households were reduced but not eliminated. Our qualitative interviews corroborated these high aspirations for education 


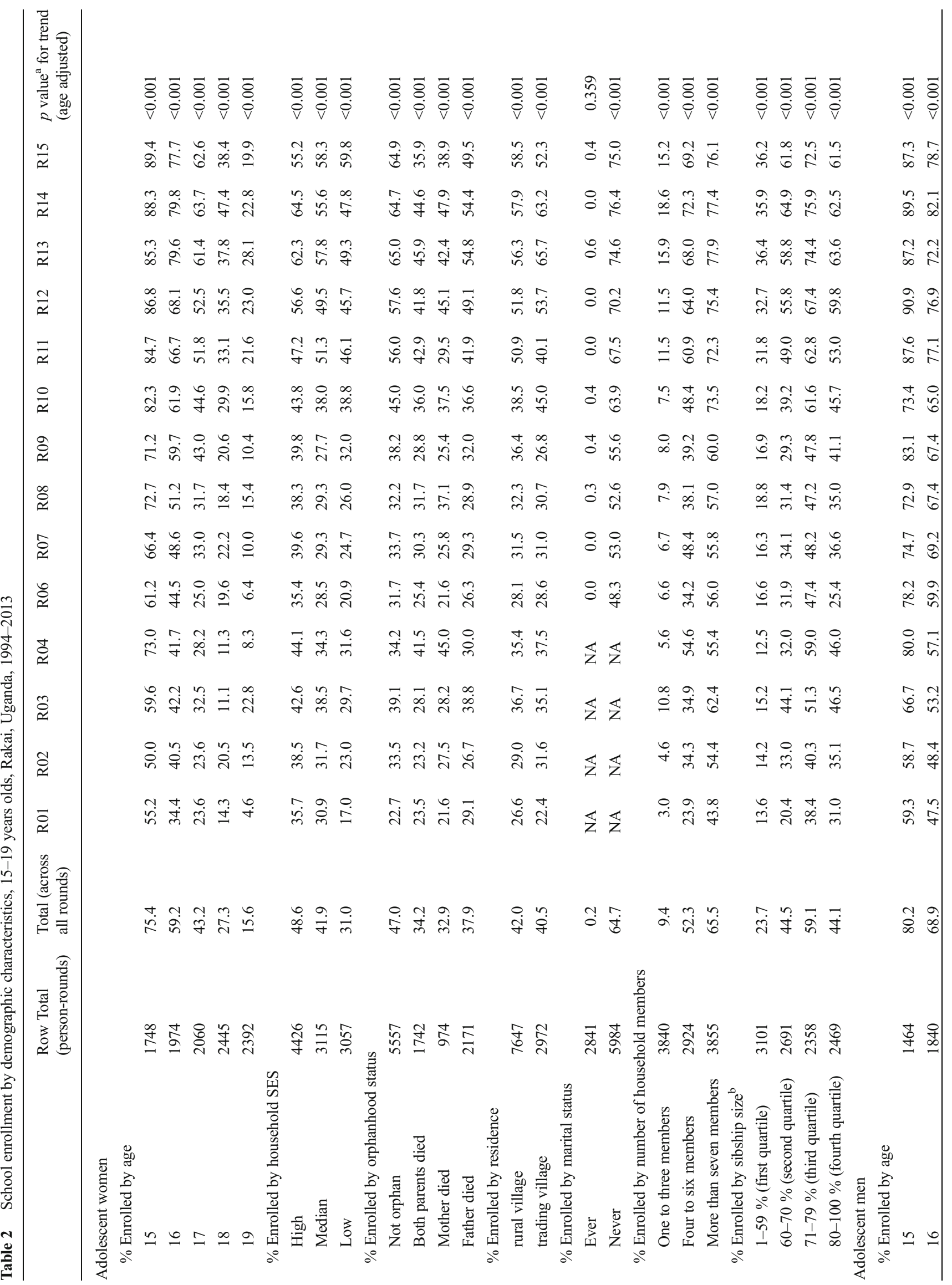




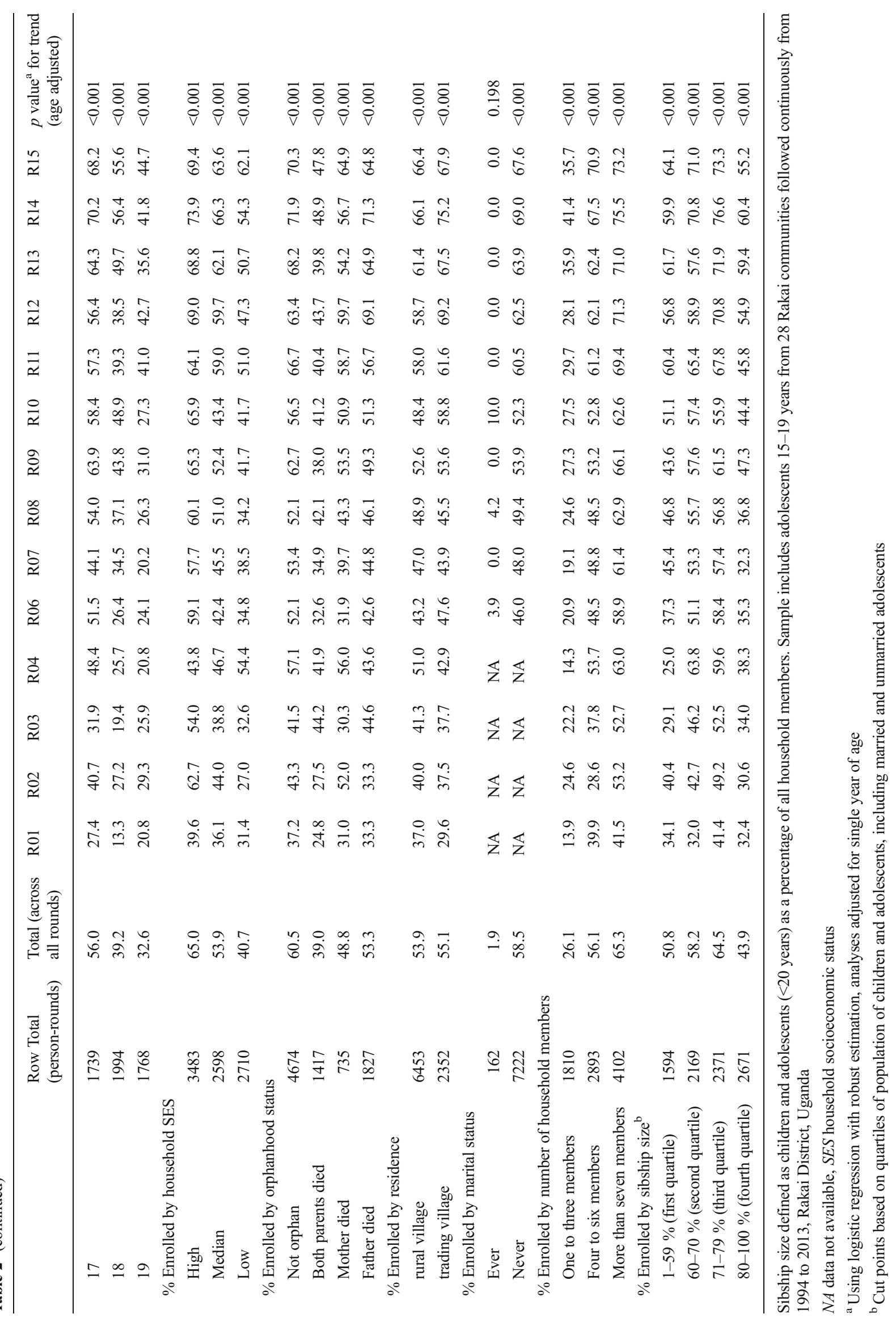




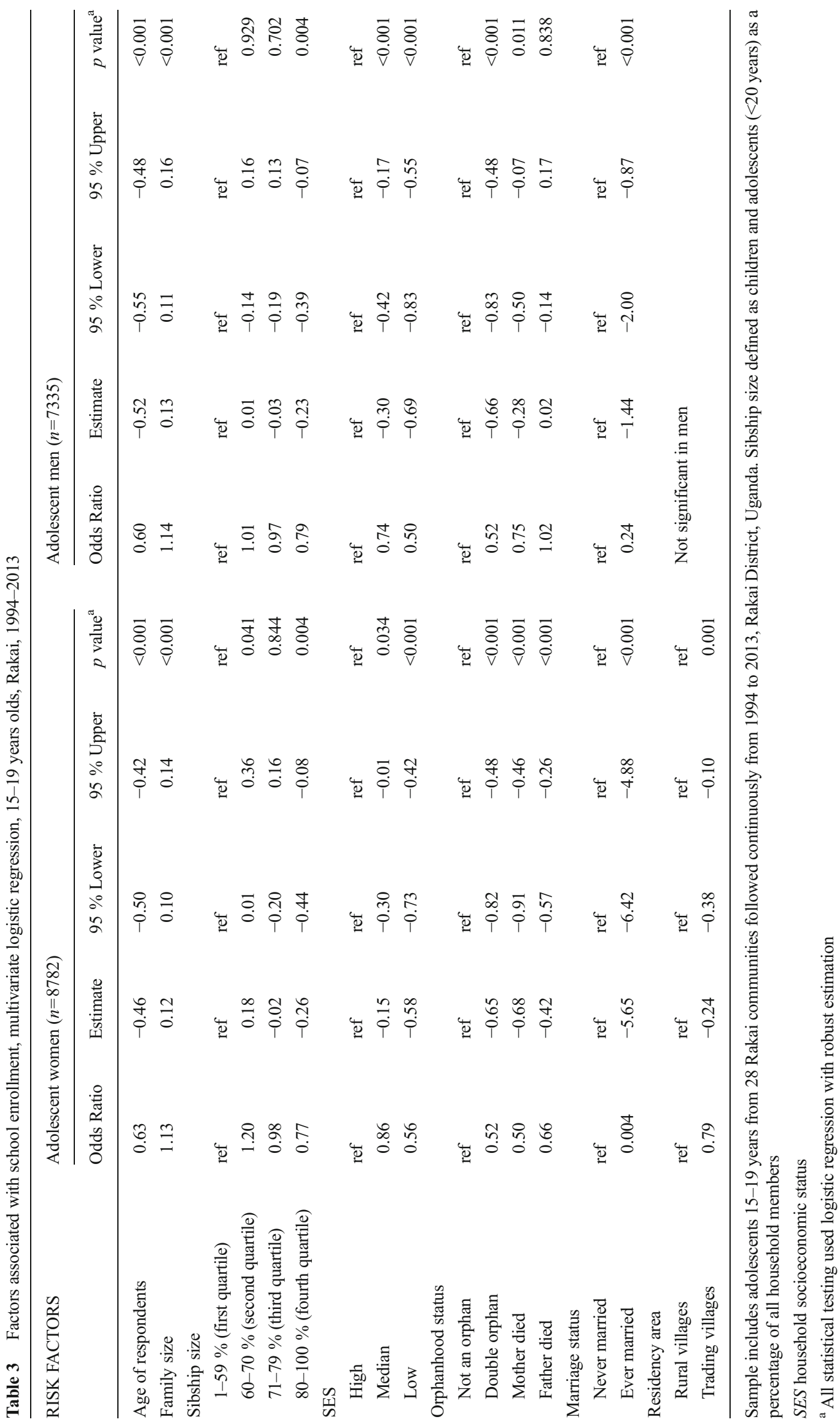




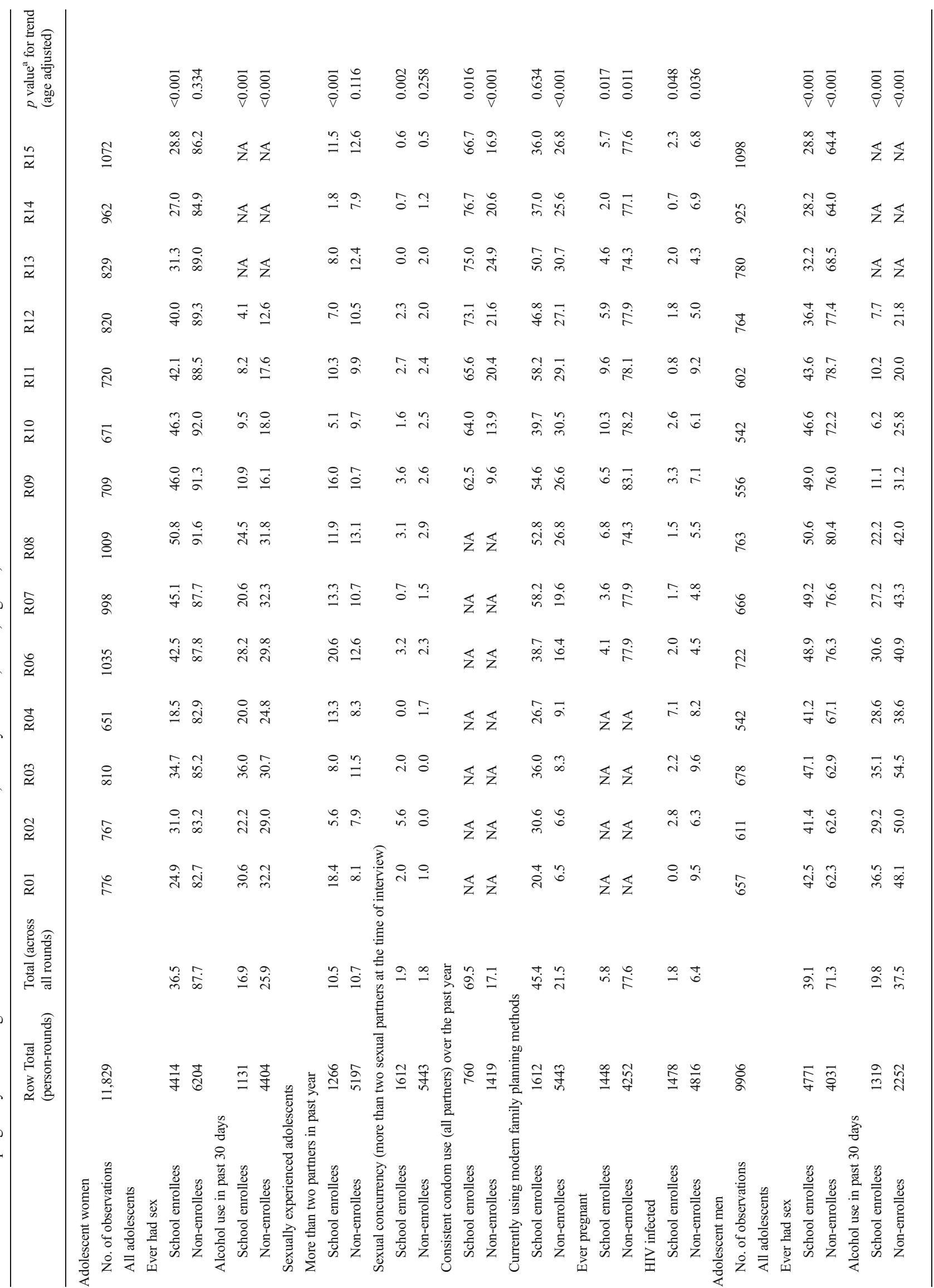


among adolescents. Likewise, we found that declining mortality among parents (as measured by adolescent orphanhood) may have increased household capacity to maintain adolescent children in school. A decline in orphanhood has also been noted among younger children in Rakai beginning around 2004 (Makumbi et al. 2012) related to the introduction of ART and reductions in HIV-related mortality among parents after 2004.

These findings reinforce the central role of schools in increasing health and well-being among adolescents (Mavedzenge et al 2013; Mmari and Blum 2009; Kirby et al. 2007; Bernat and Resnick 2006). We found that risk behaviors for HIV and pregnancy were much lower among adolescents enrolled in school; likewise, rising school enrollment was associated with declining risk behaviors for HIV and pregnancy over time. Behavior changes included lower rates of alcohol use in the past 30 days, higher rates of consistent condom use, greater use of modern contraception among women, and, among men, fewer recent sexual partners and less sexual concurrency. In prior work, school enrollment, gender, timing in sexual experience and marriage, alcohol use, and partner and relationship factors were associated with risk for HIV acquisition among youths and with trends over time in HIV acquisition (Santelli et al. 2013, 2015; Higgins et al. 2014).

In this study, gender disparities in school enrollment were reduced in the younger ages but widened amongst 18-19 years olds. For adolescent women, marriage, childbearing, and gender norms associated with educating male children might be continuing to constrain their educational aspirations.

Improvements in adolescent health associated with school enrollment can be explained in several ways. Schools may increase knowledge about HIV and pregnancy risk. Schools can also be seen as protected environments with strong social support for risk avoidance and risk reduction. Our qualitative data suggest that being a student is incompatible with social transitions such as marriage, family formation, and initiation of sexual behavior. While sex education is variable in Rakai schools, many schools support healthy adolescent transitions, risk reduction, and high aspirations via "talking compounds" using signage to promote pro-social messages at school (Iyer and Aggleton 2014, see photo).

Household size was positively associated with school enrollment among adolescents, while sibship size showed a curvilinear relationship with school. In our sample, greater household size was associated with higher SES and this may explain the association with school enrollment. We found a negative association between sibship size in the highest quartile and school enrollment; when the percentage household members under 20 years was greater than $80 \%$, the probability of school enrollment for adolescents was reduced. This association suggests that intra-familial competition to attend school 
Fig 3 School enrollment by age, women, 15-19 years, 1994-2013, Rakai District
School Enrollment by Age, Women, 15-19 years, 1994-

2013, Rakai District

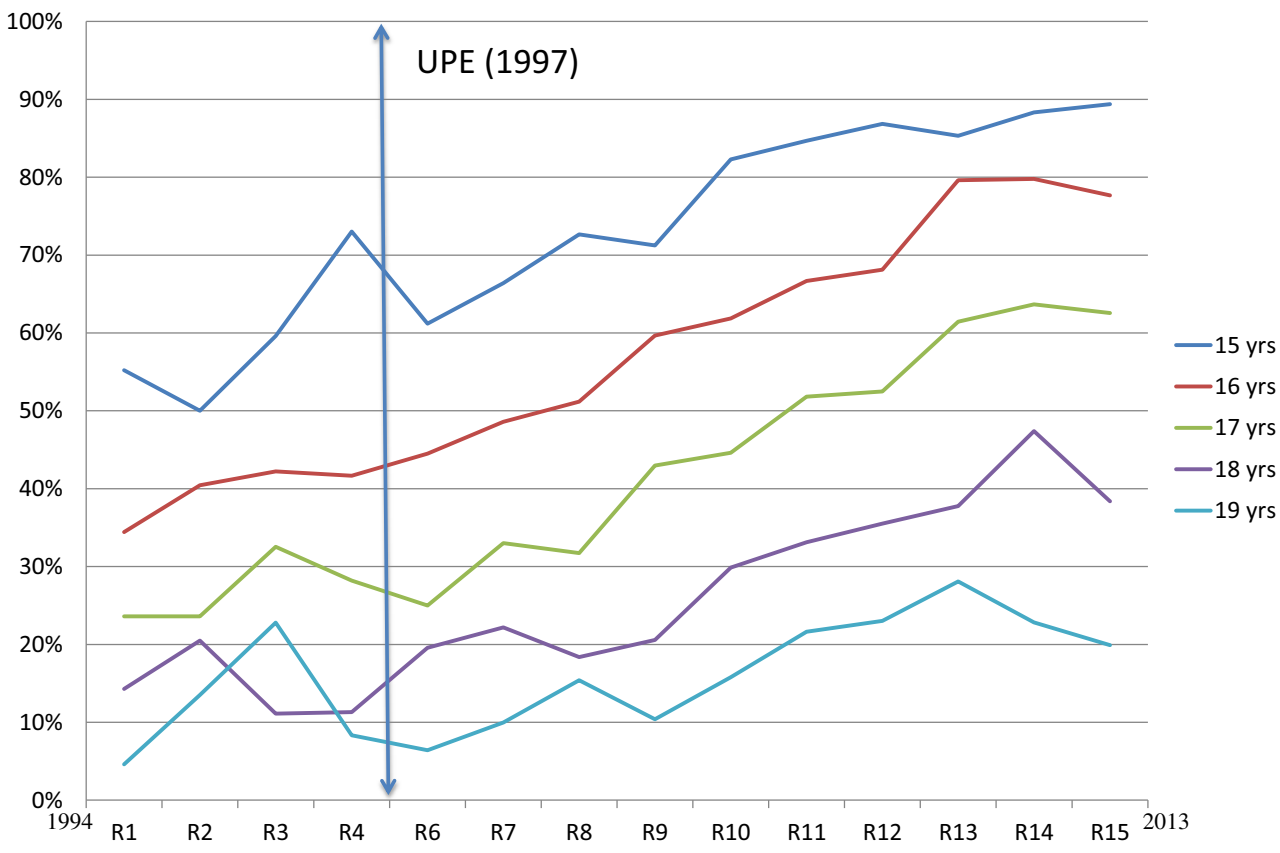

was limited to households with a high ratio of children to adults. In other social contexts, sibship size has been negatively associated with educational attainment, given household competition for educational resources (Cyrus Chu et al. 2007).

\section{Strengths and Limitations}

We note several limitations of these data. Data reported by adolescents are subject to recall and social desirability bias. For example, adolescent attending school may underreport risk behaviors because they fear repercussions by the school. However, all interviews with young people were conducted in confidential settings and not in their schools. All of the orphans in our study were living in households not institutions; the impact of living in an orphanage may be more adverse than orphanhood alone. While we were able to measure school enrollment, other factors such as the quality of education or connectedness to the school were not available for analysis. Certain data on marriage, condom use with all partners, and alcohol use were missing from some survey rounds, because data were not collected in those rounds. Finally, our study represents an examination of youths from a single rural district of Uganda; these associations may not be true in other settings.

This study also has several strengths, including the use of a population representative sample, consistent measurement of key variables over 20 years, and the ability to measure HIV serostatus. We would also note the consistency in findings between qualitative and quantitative methods.

\section{Conclusions and Implications}

School enrollment is associated with reduced risk behaviors for HIV and pregnancy among adolescents in Rakai; expanded school enrollment over the past 20 years was associated with reductions in these risk behaviors. Increasing access to schooling and efforts to retain adolescents in school could provide important improvements in adolescent sexual and reproductive health, including reduction of HIV infection.

Acknowledgments Support for this research paper and the Rakai Youth Project was provided by the National Institute of Child Health and Human Development (NICHD, 5R01HD061092-05). The authors have no conflicts of interest to report. As noted above, ethical approvals were obtained from Uganda National Council for Science and Technology and IRBs at the Uganda Virus Research Institute, Columbia University, and Johns Hopkins University. Research was performed in accordance with the ethical standards of the Declaration of Helsinki and the Belmont Report. We thank Katie Martin for her expert assistance with manuscript preparation and Margo Mullinax for her helpful editorial suggestions.

Contribution Statement John Santelli conceptualized the study, designed the data analyses, outlined the paper, wrote each draft, and coordinated each revision. Sanyukta Mathur contributed to the development of the initial paper idea, supervised data collection and analysis of qualitative data, participated in quantitative data review, and assisted in the preparation of the manuscript. Xiaoyu Song and Tzu Jung Huang cleaned the data, conducted all data analyses, participated in data interpretation, and wrote parts of the "Methods" section. Ying Wei supervised all data analyses and guided the selection of statistical methods. Tom Lutalo, Fred Nalugoda, Ron Gray, and David Serwadda provide scientific and administrative oversight to the Rakai Community Cohort Study, including the 
maintenance of the cohort and collection of data; they advised on conceptualization of the study, data analysis and interpretation, and policy implications. All authors reviewed drafts of the paper and approved the final version.

\section{References}

Bernat, D. H., \& Resnick, M. D. (2006). Healthy youth development: science and strategies. Journal of Public Health Management and Practice, 12, S10-S16.

Birdthistle, I., Floyd, S., Nyagadza, A., Mudziwapasi, N., Gregson, S., \& Glynn, J. R. (2009). Is education the link between orphanhood and HIV/HSV-2 risk among female adolescents in urban Zimbabwe? Social Science \& Medicine, 68(10), 1810-1818.

Boerma, J. T., \& Weir, S. S. (2005). Integrating demographic and epidemiological approaches to research on HIV/AIDS: the proximatedeterminants framework. The Journal of Infectious Diseases, 191(S1), S61-S67.

Caldwell, J. \& Caldwell, P. (1986). Limiting population growth and the Ford Foundation contribution.

Cleland, J. (2010). The benefits of educating women. Lancet, 376(9745), 933-934.

Cyrus Chu, C. Y., Xie, Y., \& Yu, R. (2007). Effects of sibship structure reconsidered: evidence from intrafamilial resource transfer in Taiwan. Sociology of Education, 80(April), 93-113.

Deininger, K. (2003). Does cost of schooling affect enrollment by the poor? Universal primary education in Uganda. Economics of Education Review, 22(3), 291-305.

Freedman, David, A. (2006). On the so-called "Huber sandwich estimator" and "robust standard errors". The American Statistician 60.4 .

Gakidou, E., Cowling, K., Lozano, R., \& Murray, C. J. (2010). Increased educational attainment and its effect on child mortality in 175 countries between 1970 and 2009: a systematic analysis. The Lancet, 376(9745), 959-974.

Gray, R. H., Kigozi, G., Serwadda, D., Makumbi, F., Watya, S., Nalugoda, F., Kiwanuka, N., Moulton, L. H., Chaudhary, M. A., \& Chen, M. Z. (2007). Male circumcision for HIV prevention in men in Rakai, Uganda: a randomised trial. The Lancet, 369(9562), 657-666.

Higgins, J. A., Mathur, S., Eckel, E., Kelley, L., Nakyanjo, N., Sekamwa, R., Namatovu, J., Ddaaki, W., Nakubulwa, R., Namakula, S., Nalugoda, F., \& Santelli, J. S. (2014). Importance of relationship context in HIV transmission: results from a qualitative case-control study in Rakai, Uganda. American Journal of Public Health, 104(4), 612-620.

Iyer, P., \& Aggleton, P. (2014). 'Virginity is a virtue: prevent early sex'teacher perceptions of sex education in a Ugandan secondary school. British Journal of Sociology of Education, 35(3), 432-448.
Kirby, D. B., Laris, B., \& Rolleri, L. A. (2007). Sex and HIV education programs: their impact on sexual behaviors of young people throughout the world. Journal of Adolescent Health, 40(3), 206217.

Liang, K.-Y., \& Zeger, S. L. (1986). Longitudinal data analysis using generalized linear models. Biometrika, 73, 13-22.

Lloyd, C. B. (2005). Growing up global: the changing transitions to adulthood in developing countries. National Academies Press

Makumbi, F., Nakigozi, G., Lutalo, T., Kagayi, J., Sekasanvu, J., Settuba, A., Serwada, D., Wawer, M., Gray, R. (2011). Use of HIV-related services and modern contraception among women of reproductive age, Rakai Uganda. African Journal of Reproductive Health, 14(4).

Makumbi, F. E., Nakigozi, G., Sekasanvu, J., Lukabwe, I., Kagaayi, J., Lutalo, T., Wawer, M., \& Gray, R. (2012). Incidence of orphanhood before and after implementation of a HIV care programme in Rakai, Uganda. Tropical Medicine \& International Health, 17(8), e94e102.

Mavedzenge, S., Luecke, E., \& Ross, D. (2013). Effectiveness of HIV prevention, treatment and care interventions among adolescents: a systematic review of systematic reviews. UNICEF technical brief. New York: UNICEF.

Mmari, K., \& Blum, R. (2009). Risk and protective factors that affect adolescent reproductive health in developing countries: a structured literature review. Global Public Health, 4(4), 350-366.

Pettifor, A. E., Levandowski, B. A., Macphail, C., Padian, N. S., Cohen, M. S., \& Rees, H. V. (2008). Keep them in school: the importance of education as a protective factor against HIV infection among young South African women. International Journal of Epidemiology, 37(6), 1266-1273.

Santelli, J., Edelstein, Z. R., Mathur, S., Wei, Y., Zhang, W., Orr, M. G., Higgins, J. A., Nalugoda, F., Gray, R., Wawer, M. J., \& Serwadda, D. M. (2013). Behavioral, biological, and demographic risk factors for new HIV infections among youth, Rakai, Uganda. JAIDS, 63(3), 393-400.

Santelli, J. S., Edelstein, Z. R., Wei, Y., Mathur, S., Xiaoyu, S., Ashley, S., Nalugoda, F., Lutalo, T., Gray, R., Wawer, M., \& Serwadda, S. (2015). Trends in HIV acquisition, risk factors, and prevention policies among youth in Rakai, Uganda, 1999-2011. AIDS, 29(2), 211219.

Wawer, M. J., Gray, R. H., Sewankambo, N. K., Serwadda, D., Paxton, L., Berkley, S., McNairn, D., Wabwire-Mangen, F., Li, C., \& Nalugoda, F. (1998). A randomized, community trial of intensive sexually transmitted disease control for AIDS prevention, Rakai, Uganda. AIDS, 12(10), 1211-1225.

Wawer, M. J., Sewankambo, N. K., Serwadda, D., Quinn, T. C., Kiwanuka, N., Li, C., Lutalo, T., Nalugoda, F., Gaydos, C. A., \& Moulton, L. H. (1999). Control of sexually transmitted diseases for AIDS prevention in Uganda: a randomised community trial. The Lancet, 353(9152), 525-535. 